\title{
Particle Image Velocimetry with high power Light Emitting Diodes for flow through porous medium
}

\author{
H. M. D. Harshani, S. A. Galindo-Torres \& A. Scheuermann \\ Geotechnical Engineering Centre, School of Civil Engineering, \\ University of Queensland, Australia
}

\begin{abstract}
Particle Image Velocimetry (PIV) is a non-invasive optical measurement technique which has been used for many decades to measure fluid velocity. Conventionally, PIV systems are equipped with a laser system for illuminating the flow field. However, laser systems are expensive, and their usage usually creates specific safety precautions. In this work, we used a low cost LED based illumination system in conjunction with the refractive-index matching method to measure flow velocities in porous media. Hydro jelly beads were used as a transparent porous medium which have the same refractive index as water. The movement of tracer particles which were illuminated by the LED pulse was captured by a high-speed double pulsed camera and the resulting images were processed for receiving the pore-scale fluid velocity. Interstitial velocity vectors averaged over a specified region were presented and compared with the vertical velocity component calculated from the volumetric flow rate. In general, our results demonstrated the usability of LED based PIV system for investigating flow conditions in porous media. The future aim of this study is the investigation of micro-scale seepage induced internal erosion of granular structures.

Keywords: Particle Image Velocimetry, porous flow, refractive index matching, image analysis.
\end{abstract}

\section{Introduction}

Study of flow in porous media has numerous applications such as packed column process, water flow through granular medium, blood flow through capillaries etc. 
Several experimental studies have been carried out to understand the flow and transport phenomena in porous media in macro scale which microscopic behaviour at particle scale is not measured directly. Those experiments were mainly focused on measuring average medium properties such as permeability, porosity and fluid pressure [1-3].

In the past, implementation of pore scale experiments were inherently difficult, mostly because of experimental limitations of non-invasive measurements of interstitial parameters. One major obstacle to study the mechanics of granular flow is that they are optically opaque and hence, their surface is directly visible. A most successful method to measure interstitial velocities in porous media is the use of Laser Droppler Velocimetry (LDV) in combining with Refractive Index Matching (RIM) technique. RIM is a technique which provides optical access to the bulk of the granular material by immersing particle in a fluid with same index of refraction. Budwig (1994) [4] and Wiederseiner et al. (2011) [5] provided comprehensive reviews about this technique. This makes the resulting medium transparent. Although LDV is non-invasive measurement technique, it can provide only single point velocity measurements.

Particle Image Velocimetry (PIV) technique, introduced in 1980 by Adrian [6] gives flow characteristics of entire flow field, hence, it gives a better solution for the above problem. Due to its non-invasive optical full field measurement nature, the PIV technique dominates other techniques (Adrian (2005) [6], Willert and Gharib (1991) [7]). Although these techniques are still developing, a number of excellent review papers have already been published, Lauterborn and Vogel (1984) [8], Adrian (1986, 1991) [9, 10], Grant (1997) [11] and Prasad (2000) [12], which together describe the historical development, measurement technique, and applications of PIV. PIV has been used in combination with RIM to measure interstitial velocity in porous medium by Northrup et al. (1993) [13]. In PIV experiments, it is necessary to seed the fluid with tracer particles which follows the motion of the fluid. To measure the flow velocity, the interested area is illuminated at least twice within a short time interval by means of a light sheet. It is assumed that seeding particles follow the fluid velocity between these two illuminations. The light scattered by the particles is recorded on a single frame on a sequence of frames. Velocity field is then calculated by measuring the distance travelled by the particles during the time between light pulses.

In most PIV experiments, laser is used as the light optics to illuminate the seeded flow. However, it is very expensive to use laser based PIV and their usage usually creates specific safety requirements. Currently, there is a trend towards, designing cost effective PIV systems. Light Emitting Diodes (LED) have number of attractive features like low cost, low power consumption and high brightness which are necessary for PIV experiments (Willert et al. (2010) [14], Buchmann et al. (2012) [15]). The literature reports the use of LEDs for bubble shadowgraphs $[16,17]$ shadow velocimetry [18] and for PIV [14].

Estevadeordal and Goss [18] have demonstrated LED illumination PIV with shadows of particles in air-flow [19]. They also demonstrated measurements in pulse mode and supplied a discussion on some general considerations for PIV 
with LEDs. Chételat and Kim (2002) [19] have developed a low-cost miniature PIV system with LED illumination in a forward and backward scattering mode. This system has the capability of measuring $10 \mu \mathrm{m}$ particles with $1 \mathrm{~ms}^{-1}$ in water and $50 \mu \mathrm{m}$ droplets with $10 \mathrm{~ms}^{-1}$ in air. Willert et al. (2010) [14] investigated high power pulsed operation LEDs for measuring flow characteristics using side scattering technique.

This paper presents a development of a LED based PIV system which can be used to quantify the flow field and validation of the PIV results were carried out with volumetric flow rate measured by a flow meter. In section 3, development of the experimental setup to measure flow fields including transparent porous structure and imaging system is described. Length calibration, pulsed delay study, which are important pre processing procedures, are described here. Results of the measurements are presented in section 4 . The conclusion of the paper is given at section 5 .

\section{Experimental setup and methodology}

Fig. 1 shows a schematic diagram of the experimental setup. It consists of a constant head tank, flow meter, pump, test section, LED light head, high speed camera, synchronizer, seeded flow and solid particles. RIM and PIV techniques were used to capture the fluid flow at micro scale. Each part of the experimental setup is described in the next sections.

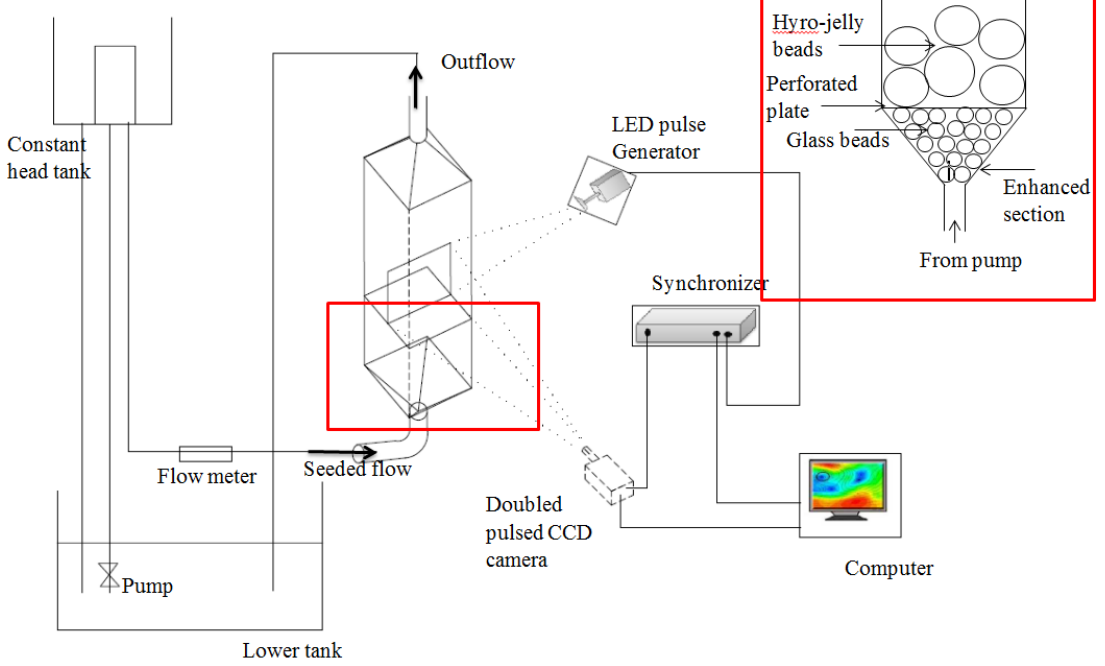

Figure 1: Schematic arrangement for Particle Image Velocimetry system. 


\subsection{Flow system}

Test section which has the cross section of $100 \mathrm{~mm} \times 100 \mathrm{~mm}$ with $300 \mathrm{~mm}$ height, was designed for the experiment as in fig. 2(a). The conical shape of the entrance of the channel assures a uniform flow and pressure at the inlet of the bed. Glass beads were filled to this section to further assure a relatively uniform inlet flow condition. The bed was filled from hydro-jelly beads with $7 \mathrm{~mm}$ diameter in a random manner. Constant head tank was used to introduce a steady flow rate while also allowing for adjustment of the hydraulic head. Fluid was collected to the lower tank and pumped back to the constant head tank. Flow rate through the channel was measured through a flow meter connected to the system and flow was controlled through a valve. RIM media was achieved by using hydrojelly beads as a solid skeleton which has the same refractive index as water. This makes the resulting medium transparent and possible to use water as fluid phase. Tracer particles play a key role in indoor PIV measurements, because PIV actually

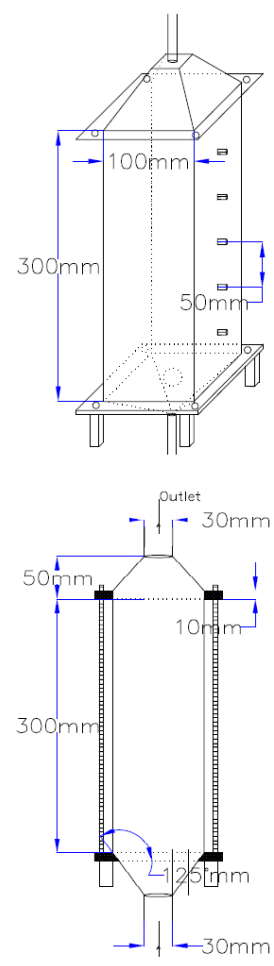

(a)
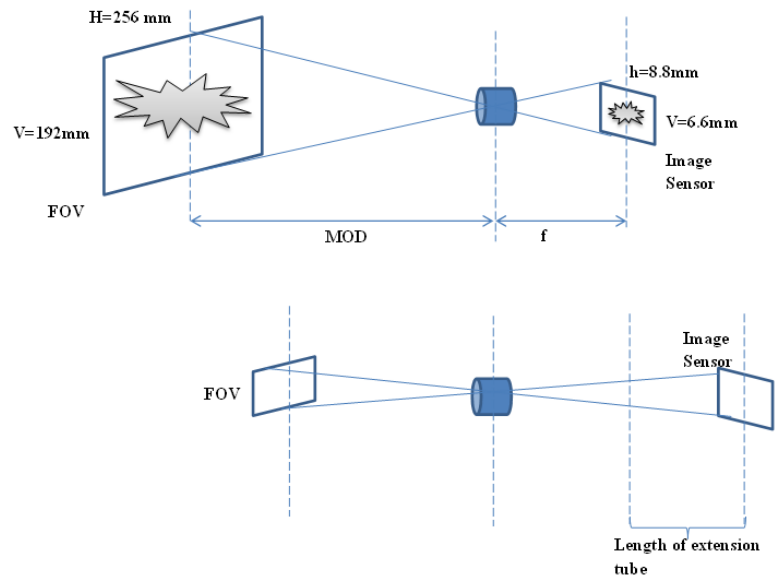

(b)

Figure 2: (a) Schematic drawing of the test section which is made by acrylic, (b) FOV changes with the original sensor and with the extension tube. 
measures the velocities of tracer particles instead of real flow velocities. Flow was seeded with $20 \mu \mathrm{m}$ diameter Polyamide Seeding Particles (PSP). It is important to maintain an uniform seeding throughout the experiment. Particle density of the flow was maintained at $20 \mathrm{mg} / \mathrm{l}$ which has acceptable seeding concentration for images. These seeding particles have a density of $1.1 \mathrm{~kg} / \mathrm{m}^{-3}$ and those are naturally buoyant. The gravitationally induced velocity, $\mathrm{U}_{g}$, can be derived from the Stokes' drag law (eqn. (1)), which is considered to apply when using the assumption of spherical particles in a viscous fluid at a low Reynolds number. This condition holds true for most indoor PIV applications.

$$
U_{g}=d_{p}^{2} \frac{\rho_{p}-\rho_{f}}{18 \mu} g
$$

where $g$ is the gravitational acceleration, $\rho_{f}$ and $\mu$ are the density and dynamic viscosity of water, $\rho_{p}$ and $d_{p}$ are the density and diameter of the particles.

\subsection{LED based PIV system}

The basic principle of Particle Image Velocimetry (PIV) is to capture a pair of images of the flow under study with a specific time interval between them. The measured small difference in particle positions between images is used to compute for the velocity flow field. High power LED pulsing system provided by Intelligent Laser Applications (ILA GmbH) was used as light source. LED head was connected to a fibre coupled optics in order to have a thin light sheet. Plane illumination was achieved by LED device of $25 \mu$ s duration and pulse delay $(\delta \mathrm{t})$ was set according to the flow velocity. Images were recorded using high speed double shutter camera from PCO imaging. The camera has a 14 bit sensor with $1392 \times 1040$ pixels. Two images with $1 \mu$ s inter framing time can be recorded in double shutter mode. $50 \mathrm{~mm}$ focal length with c mount type lens was used for the experiment. To have a larger magnification $10 \mathrm{~mm}$ length extension tube was set between the lens and the sensor (fig. 2(b)). Synchronizer was connected to a personnel computer and driven by Labview software. It controls the timing between camera frame and light pulse duration. Final PIV settings adopted in this study are summarized in table 1 .

The Field of View (FOV) had dimensions of $47 \mathrm{~mm} \times 35 \mathrm{~mm}$. Because the images were composed of $1392 \times 1040$ pixels, spatial resolution per pixel is therefore $33.7 \mu \mathrm{m}$. PIV in itself provides velocity in pixels per second. To convert to physical space velocity, the true physical dimensions of the image pixels must be known. For this, vertical and horizontal length calibration were done.Prior to PIV image precessing, the images were improved by applying a high pass and low pass filter during the post processing step. Fig. 3(a) and 3(b) shows a raw image before the pre-processing analysis and improved image respectively. 


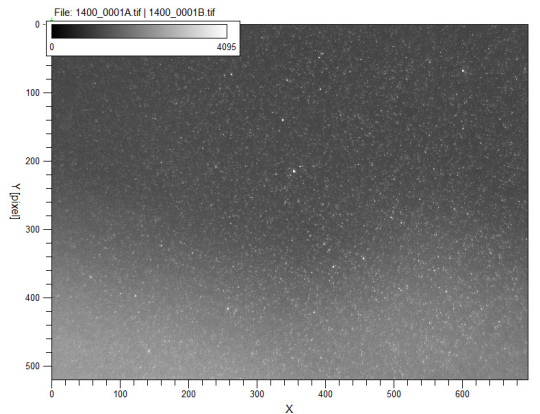

(a)

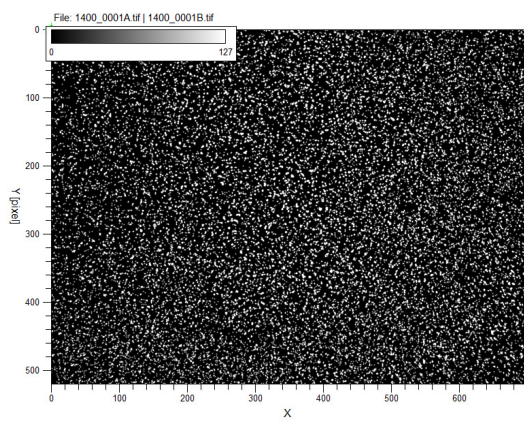

(b)

Figure 3: (a) Raw image obtained from the PIV imaging system, (b) image which is improved by pre-processing analysis.

Table 1: Particle Image Velocimetry (PIV) system settings used for the experiment.

\begin{tabular}{|l|l|l|}
\hline Parameter & Value & Unit \\
\hline Resolution & $1392^{*} 1040$ & Pixel \\
\hline Pixel size & $6.45^{*} 6.45$ & $\mu \mathrm{m}$ \\
\hline sensor format & $2 / 3$ & $\mathrm{fps}$ \\
\hline Frame rate & 7.3 & $\mathrm{fps}$ \\
\hline Exposure time & $5 \mu \mathrm{s} . . .60 \mathrm{~s}$ & \\
\hline Interframing time & 1 & $\mu \mathrm{s}$ \\
\hline
\end{tabular}

\section{Results and discussion}

\subsection{Image processing}

Each image was first pre-processed to remove the background and electronic noise in order to improve the quality. The noise removal was achieved by using grey level thresholding (both high and low pass filtering. Fig. 3(b) shows the improved image. The process of identification of the particle images is then begun. For identifying particle patterns, PIVview2C-software (PIVTec GMBH, Germany) was used. Image area was divided into interrogation spots of $24 \times 24$ pixels, and the movement of each spot was calculated using the 'multigrid cross correlation' 
method. In this method, each spot at time step $\delta \mathrm{t}$ is moved in the $x$ and $y$ direction (up to a maximum of 12 pixels) and simultaneously rotated around its center. The distance between the starting point and the point of highest probability of overlap with the image data of time step $\delta \mathrm{t}+1$ is taken as a velocity vector. To remove poorly correlated vectors, a moving average filter with a $3 \times 3$ window was applied on the cross- correlated images. Fig. 4 shows the velocity field and contour plotting of a sample image from the preliminary analysis.

\subsection{Time delay $(\delta \mathrm{t})$ between pulses}

The time interval between image acquisitions is a critical parameter because there must be sufficient movement of the tracer particle within the interrogation window to accurately represent the flow velocity. The time delay $\delta$ t should not only be long enough to determine the displacement of the tracer particles between the two pulses, but also need to be short enough to avoid particles leaving out of the light sheet between subsequent illuminations. Fig. 5 shows velocity fields obtained by image analysis for three different $\delta$ t values.

The high number of rejected vectors is due to the particles that have already exited the window in the second image or new particles have entered into the window in the second image. Hence, it is important to find the correct $\delta$ t value for different velocities of the flow. For this study $2 \mathrm{~ms}$ of pulse delay is used for $0.016 \mathrm{~ms}^{-1}$ flow velocity.

\subsection{Results verification}

PIV results were verified with measured volumetric flow rate given by the flow meter by having only liquid flow inside the section. Flow velocity $\left(V_{i}\right)$ for each section along $Y$ direction as shown in fig. 6 were obtained and calculated the

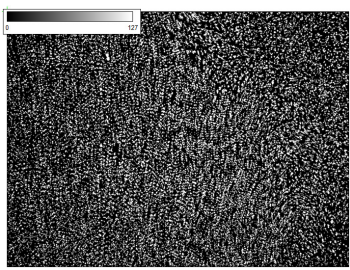

(a)

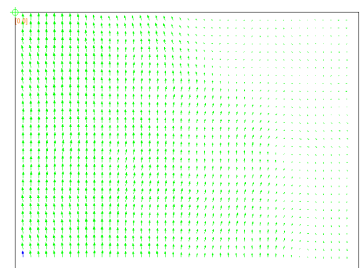

(b)

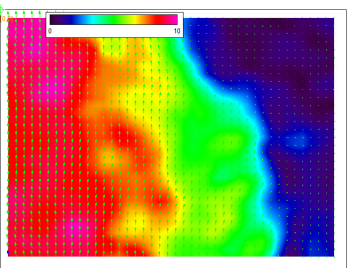

(c)

Figure 4: (a) image data of preliminary test, (b) velocity field corresponding to the image, (c) contour plot for the velocity. 
average velocity $\left(V_{\text {aver }}\right)$ by eqn. (2),

$$
V_{\text {aver }}=\frac{\sum_{i=1}^{10} V_{i} d_{x} d_{y}}{A},
$$

where $A$ is total area of test section, $d_{x}$ is length of test section in $X$ direction and $d_{y}$ is the width of the considered strip in $Y$ direction. Ten strips were considered along $Y$ direction. Volumetric flow rate (Q) was measured from flow meter and used to calculate average velocity $\left(V_{\text {aver }} \mathrm{FM}\right)$ which was calculated to be $0.0344 \mathrm{~ms}^{-1}$. The corresponding PIV analysis, $V_{\text {aver }} \mathrm{PIV}$, was $0.0356 \mathrm{~ms}^{-1}$. Thus the estimated error between the mean velocity measurements obtained using the flow meter and PIV is approximately $3.5 \%$.

\subsection{Porous medium flow}

After having successful validation, the system was used to capture the flow behaviour though pore structure. Planar sections of two component velocity data were taken at the middle of the bed. Fig. 7(a) shows a representative double exposure PIV raw image obtained from the porous medium bed. and improved

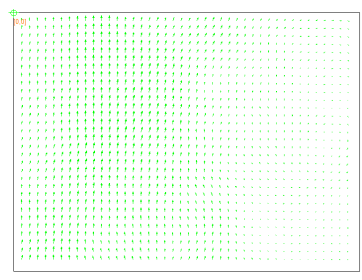

(a)

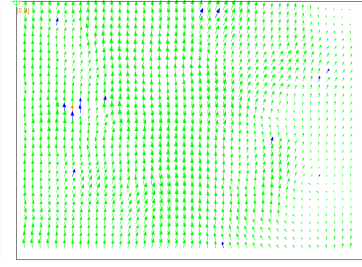

(b)

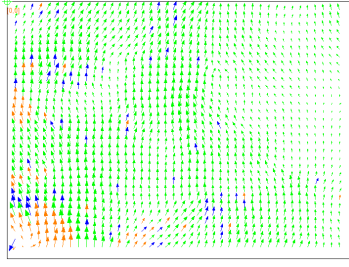

(c)

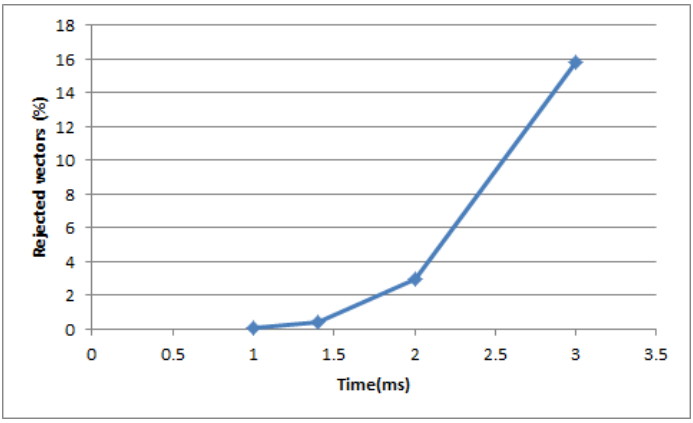

(d)

Figure 5: Effect of the $\delta \mathrm{t}$ for velocity field (a) $\delta \mathrm{t}=1 \mathrm{~ms}$, (b) $\delta \mathrm{t}=2 \mathrm{~ms}$, (c) $\delta \mathrm{t}=$ $4 \mathrm{~ms}$, (d) variation of the percentage of rejected vectors with increasing of $\delta \mathrm{t}$. 

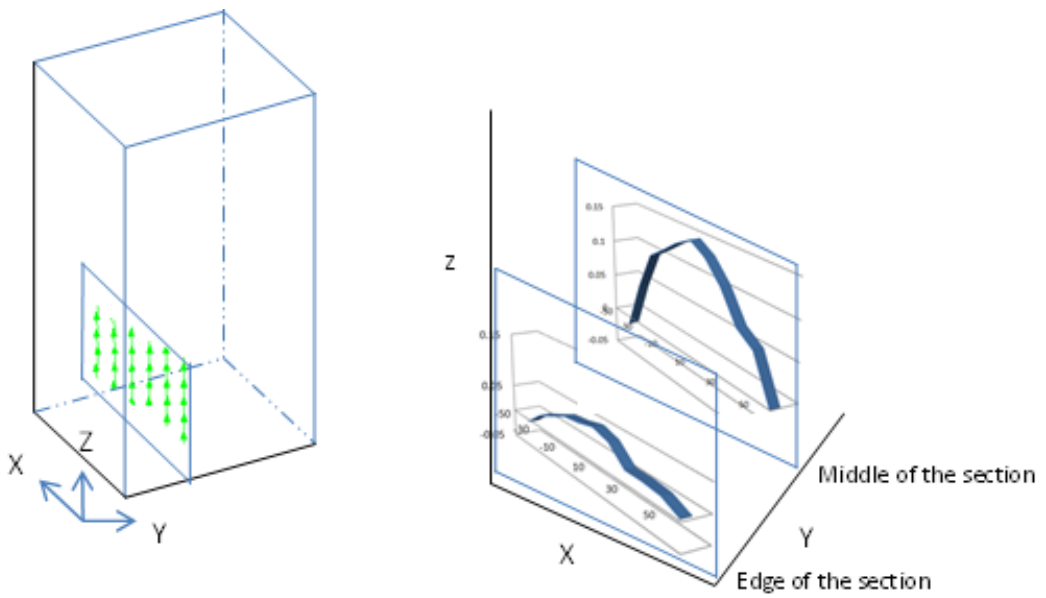

Figure 6: Comparison of PIV results with flow profile along the test section.

image is shown in fig. 7(b). Bright spots are images of seeding particles and dark round areas correspond to the location of hydro-jelly beads which represents the porous structure. Various pores were scanned using preliminary results to help identify initial characteristics that differ from pore to pore. These investigations are just at the beginning which is why only an image can be presented in this paper.

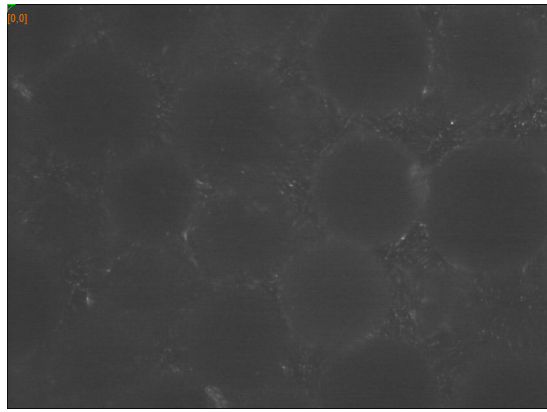

(a)

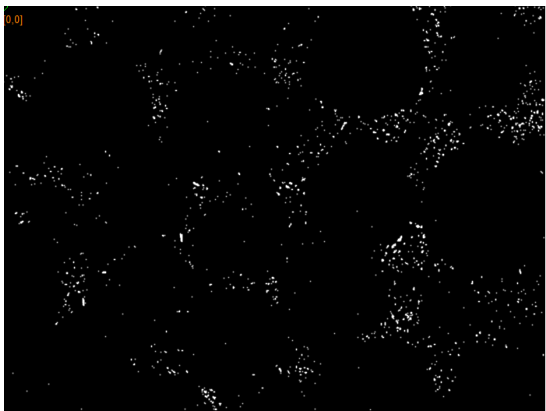

(b)

Figure 7: (a) Preliminary image of porous flow though hydro-jelly beads, (b) Porous flow image improved through filtering procedure. 


\section{Conclusions}

In this study we have investigated that viability of using pulsed, high-power LED as a illumination source for PIV measurements. Classical side scattering imaging configurations has been used to illuminate the flow filed. System was used to validate the PIV system for quantifying the flow field. Although LED based PIV systems have been frequently used in the micro-PIV measurements in the literature, our system shows the capability of measuring $47 \mathrm{~mm} \times 35 \mathrm{~mm}$ FOV by using $20 \mu \mathrm{m}$ seeding particles. Results show that high power LED devices are very promising alternative to flow visualization instead of high cost laser systems.

\section{Acknowledgement}

The presented research is part of the Discovery Project (DP120102188) Hydraulic erosion of granular structures: Experiments and computational simulations funded by the Australian Research Council.

\section{References}

[1] Bear, J. \& Bachmat, Y., Transport phenomena in porous media: basic equations. pp. 3-61, 1984.

[2] Schwartz, C.E. \& Smith, J., Flow distribution in packed beds. Industrial \& Engineering Chemistry, 45(6), pp. 1209-1218, 1953.

[3] Skempton, A. \& Brogan, J., Experiments on piping in sandy gravels. Geotechnique, 44(3), pp. 449-460, 1994.

[4] Budwig, R., Refractive index matching methods for liquid flow investigations. Experiments in Fluids, 17(5), pp. 350-355, 1994.

[5] Wiederseiner, S., Andreini, N., Epely-Chauvin, G. \& Ancey, C., Refractiveindex and density matching in concentrated particle suspensions: a review. Experiments in Fluids, 50(5), pp. 1183-1206, 2011.

[6] Adrian, R.J., Twenty years of particle image velocimetry. Experiments in Fluids, 39(2), pp. 159-169, 2005.

[7] Willert, C.E. \& Gharib, M., Digital particle image velocimetry. Experiments in Fluids, 10(4), pp. 181-193, 1991.

[8] Lauterborn, W. \& Vogel, A., Modern optical techniques in fluid mechanics. Annual Review of Fluid Mechanics, 16(1), pp. 223-244, 1984.

[9] Adrian, R., Multi-point optical measurements of simultaneous vectors in unsteady flow: a review. International Journal of Heat and Fluid Flow, 7(2), pp. 127-145, 1986.

[10] Adrian, R.J., Particle-imaging techniques for experimental fluid mechanics. Annual Review of Fluid Mechanics, 23(1), pp. 261-304, 1991.

[11] Grant, I., Particle image velocimetry: a review. Proceedings of the Institution of Mechanical Engineers, Part C: Journal of Mechanical Engineering Science, 211(1), pp. 55-76, 1997. 
[12] Prasad, A.K., Particle image velocimetry. Current Science-Bangalore, 79(1), pp. 51-60, 2000.

[13] Northrup, M.A., Kulp, T.J., Angel, S.M. \& Pinder, G.F., Direct measurement of interstitial velocity field variations in a porous medium using fluorescentparticle image velocimetry. Chemical Engineering Science, 48(1), pp. 13-21, 1993.

[14] Willert, C., Stasicki, B., Klinner, J. \& Moessner, S., Pulsed operation of high-power light emitting diodes for imaging flow velocimetry. Measurement Science and Technology, 21(7), p. 075402, 2010.

[15] Buchmann, N.A., Willert, C.E. \& Soria, J., Pulsed, high-power led illumination for tomographic particle image velocimetry. Experiments in Fluids, 53(5), pp. 1545-1560, 2012.

[16] Bröder, D. \& Sommerfeld, M., Planar shadow image velocimetry for the analysis of the hydrodynamics in bubbly flows. Measurement Science and Technology, 18(8), p. 2513, 2007.

[17] Nogueira, S., Sousa, R., Pinto, A., Riethmuller, M. \& Campos, J., Simultaneous PIV and pulsed shadow technique in slug flow: a solution for optical problems. Experiments in Fluids, 35(6), pp. 598-609, 2003.

[18] Estevadeordal, J. \& Goss, L., PIV with LED: particle shadow velocimetry (psv). 43rd AIAA aerospace sciences meeting and exhibit, meeting papers, pp. 12355-12364, 2005.

[19] Chételat, O. \& Kim, K.C., Miniature particle image velocimetry system with led in-line illumination. Measurement Science and Technology, 13(7), p. 1006, 2002. 